\title{
A Literature Survey on Methodologies for Classification, Maturity Detection, Defect Identification and Grading of Fruits
}

\author{
Reshma R. \\ Dept. of Computer Science \\ College of Engineering Poonjar
}

\author{
Sreekumar K. \\ Dept. of Computer Science \\ College of Engineering Poonjar
}

\begin{abstract}
The agricultural sector is the primary and unavoidable sector in Kerala .Kerala is a green state in here wide variety of trees and plants are present. There are different species of trees and few of them are dig for fruits. Fruit has been accepted as a good source of vitamins, minerals and fibers. Most commonly used fruits are mongo, jack fruit, banana etc. This work gives as the review of the fruit Classification, grading, maturity identification and defect detection. Image acquisition performed with digital camera. Fruits are classified based on different features like size, color, texture, etc ... the presence of defects on the fruits affects the market value of the product. Now fruit quality estimation using machine vision in Kerala is ongoing.
\end{abstract}

\section{Keywords}

Artificial Neural Network, Fuzzy logic, Grading

\section{INTRODUCTION}

Kerala is known as gods own country because of lots of trees, river, and paddy fields. Agriculture is the primary food source of Kerala. Fruits are the one of large groups of food production that carries the antioxidant. Fruits and vegetables are the vital part of the food and highly nutritious.

Computer vision and image processing in agriculture are the two major fast growing research areas. The aim at this review paper is to research the use of image processing and computer vision techniques in the food industry mainly in fruit classification grading maturity identification and defect detection

Classification or categorization is the process of recognition of objects. Classification in machine learning use different classifiers mainly used classifiers is supporting vector machine nearest neighbor classifier, Fuzzy system, Artificial Neural Network

Grading is the process of sorting the data with respect to the parameters such as dimension. Grading is the most essential step used in the post-harvesting of fruits. The fruit grading system based on image processing used different types of algorithms for detecting and sorting through fruits. The case of international market generally 3 grades are used for fruits and vegetables, they are Extra class, Class 1 and Class 2.the main advantage of the grading are avoiding the heavy packing cost and transportation rate Morphological and color features are extracted using neural net work, fuzzy logics, and some other algorithms. The captured images are in RGB format, for our purpose we can convert into another color format. [15] Say that using eccentricity, extend and cross ratio properties. of the shape classify the mango fruit of well formed or deformed. The result of these values fed to the fuzzy system for the classification

\section{LITERATURE SURVEY}

From the evidences we found that the use of fruits may decrease the cardio vascular dieses and cancer. Pre processing and feature extraction is the two common process of Classification, grading, maturity identification and defect detection.

\subsection{Pre-processing steps}

In all works considered for review, preprocessing has been the essential first step of the for classification, grading, maturity identification and defect detection activities. The fruits were captured using digital camera either set up an image capturing system or shot directly. The pre-processing technique standardizes the orientation and scale of the captured images. The main methods found are,

\subsubsection{RGB to gray conversion}

In [1] Defined RGB to gray conversion is a low level preprocessing. Mainly two methods are used for RGB to GRAY conversion. They are average method and weighted method. Average method is very simple and average of $R, G$, and $\mathrm{B}$ is taken.

\subsubsection{Binary conversion}

Binary images also known as Digital images. Possible values of the images are 0 and 1.color of the images is either black or white. In [7] Says main clinical part of the system is Binary conversion using ostu's algorithm.

\subsubsection{Noise reduction}

Noise is the unwanted things in the image. For noise reduction majority of them are using average filter [7]. In [9] split and merge algorithm is used for the noise reduction

\subsubsection{Contrast stretching}

It is a simple image enhancement process. Using contract stretching method improves the quality of the images. In the case of color images are stretched using the same offset and scaling to preserve the correct ratios of color.

\subsubsection{Histogram equalization}

Using histogram we can find the picture element with possible gray scale value. Contrast enhancement of image done by adjusting the intensities. Range of the intensity of pixels is 0 to $1-1$. L is the possible intensity. Initially read the image then evaluates the histogram then obtained normalized sum finally transforms into output. 


\subsubsection{Background elimination}

Removal of unwanted areas in the image is taking place. Yudong zhang (2016) reported split and merge algorithm is commonly used for background elimination and square window for foreground detection

\subsection{Feature extraction}

Feature extraction is an initialization from the measured data and derived the features of the images. In here the different features extracted from the fruits. This section gives an idea of different features used for the operation. Mainly used features are

\subsubsection{Shape}

Dameshwari sahu describe shape description include the Region and boundary. The grid based and moment approaches are suitable for region description.

\subsubsection{Size}

Fruit size measured from the area and volume of fruits. The size of the fruit increases by the use of nutrition.

\subsubsection{Texture}

Date fruit classification using texture descriptors and shape size features $-2014[17]$ by ghulam Muhammad says that the texture descriptors are in the form of Local Binary Pattern (LBP) or WLD. The result obtained from the system is better for date fruit classification. Texture is distinguished by entropy.

\subsubsection{Color}

$\mathrm{RGB}, \mathrm{YCbCr}$ and $\mathrm{HSV}$ are mainly used three types of color spaces. Colors of the fruits are very useful for the ripening prediction. The color images stored memory are called 'Raster Maps'

In color segmentation process range of in HSVis identified and Converted pixels to Array [23]

Morphological operation erosion and dilations are used for elimination of small objects. [23].Dilation is the summation of pixels to the boundary and erosion is the abolishment of pixels on the edges is takes place. Adding and removing of pixels is based on the shape and size of structuring element.

\subsection{Classification Methods}

For the classification of fruits different types of classifiers are used. Mainly used classifiers are briefly describe below

\subsubsection{Fuzzy logic}

It is the one of the famous classification algorithms in modern era. It is based on if-then rule. Classify the mango into small; large and medium using fuzzy logic .fuzzy logic is performed by using matlab tools [11].it handles the uncertainty and ambiguity of the classification. The main advantage of fuzzy logic allow natural description, it solved the relationship between the numerical values. It is a branch of logic designed for the representation of knowledge. It has different degree of membership

\subsubsection{Artificial Neural Network}

$\mathrm{NN}$ is Very useful computational model. Working of $\mathrm{NN}$ is similar to the human neurons. The $\mathrm{NN}$ is easily managing the complex problems. It gives high accuracy.Emny Harna Yossy [23] reported mango fruit sortation system using $\mathrm{NN}$ and computer vision- the first step of the system is preprocessing. In that time captured RGB value format converted into HSV format. Neural network to be built is a multicultural network with symmetrical sigmoid activation function and with back propagation training. Mangoes are sorted into 4 classes. In here prediction of color and size takes place with NN.There are 40 hidden layers for this work. The system obtained $94 \%$ accuracy. [9] Describe FNN did not need any prior knowledge of the images.

\subsubsection{Support vector machine}

Classification of data is the common process of machine learning.SVM used the separation of points using hyper plane. Accuracy of the SVM is higher than other classifiers. It is a supervised learning.

\subsubsection{K-Nearest Neighbor}

It is mostly used for classification and regression. Output of the classifier is a class membership. It is a type of lazy learning algorithm

\subsection{Review of machine learning tasks related to fruits}

\subsubsection{Grading}

[2] Suggest the automated grading of mango with the help of maturity detection. The proposed system uses the RGB to HSV conversion algorithm and k-means for the grading of harvested mango. Five verities of mango graded in here and overall accuracy of the system obtained is $84 \%$.

Mohammad A.H.Ali in his work automated fruit grading system -described he used two kinds of fruits are used for processing they are apple and mango. Grading is carried out using MATLAB software features like size; shape colors are improving the accuracy of this work. The boundary of the fruit identified using canny edge detection. He says that automatic grading of fruits is comparatively easy than manual grading.

[8] In here grading is dome with computer vision. In here grading is performed with color. Initially sRGB color converted to $\mathrm{L}^{*} \mathrm{a} * \mathrm{~b}$. then found pixels in red area using threshold. The region of interest plotted on chroma-hue angle.CVS is very useful for finding Anthocytocin concentration. Ripped fruits of higher Anthocytocin, the highest red color indicated the ripped stage of fruit. Grading with ripening is done accurately.

Scientific classification of ripening period and development of color grade chart for Indian mangoes using multivariant cluster analysis by V.Eyar et al. (2015) [14] used two types of mango Banganapally andAlphonso for grading and calculation of ripening period. Color format for mango grading is $\mathrm{L}^{*} \mathrm{a}^{*} \mathrm{~b}^{*}$, calculated using Hunter Lab Color meter. From this he observed ripening of Alphonso need 19 days but Banganapally have 23 days. Total soluble solids of mangoes increase linearly to 16th day after that there is no change obtained In Alphonso. In here mango classified into 5 they are unripe, early ripe, partially ripe, ripe and decay. The best stage for packing 1 and 2 in the case of Alphonso, it is 1-6 days and case of Banganapally is 1-8.

In [11] the author grades the mango fruit using fuzzy logic. Classify the mango into small, medium and large. The result of the system obtained is mango with size $<=30$ consider as small; between 30-50as medium greater than 51 is large mango. The accuracy of the system is $85 \%$

Shape and weight grading of mangoes using visible image by M.F Ibrahim (2015) proposed cylindrical method is best method for weight calculation of mangoes using images.RGB to HSI transformation is highlighting of this work. Grading of 
mango with SVM got $100 \%$ accuracy and accuracy of DA is 98.3\%.Descriminent analysis identifies the subset of the dominant features then split it into different groups. Cylinder approximation analysis achieves 95\% in the case of weight calculation of mangoes.

\subsubsection{Maturity identification}

$\mathrm{RGb}^{*}$, grayscale and $\mathrm{S}$ channels values are used for find different between maturity stages. The length and aspect ratio is doing not to affect the ripeness of fruits. In here the fruit classified into three based on maturity they are unripe, ripe, and overripe. The analysis of variance performed using SPSS software. The overall accuracy of the system obtained is 90.24\%. Classification performed using LDA and QDA, the accuracy level is higher in the case of QDA [21]

In [31] define Mango fruit maturity detection is done using its external color. Special species mangos "Langdo" had the same color in its life span. In here x-ray, infrared are used and photos of mango taken using thermal camera. Images are in $\mathrm{L} * \mathrm{a} * \mathrm{~b} *$ format. $89 \%$ accuracy is evaluated in here

\subsubsection{Defect detection}

In [6], images are captured using video and divide it into 100 frames. Bacterial affected area of mango are separated using threshold value and grading is based on affected area in here watershed algorithm is used for the bacterial affected area identification. By using this method accurately classified and detected the external defects of mango fruit

Color, texture, size, a shape is used for inspection of fruits and vegetables. Region oriented segmented algorithm identify the peel defects of citrus fruit using computer vision [28]

Automatic classification of fruit defect based on cooccurrence matrix and Neural Network (2015) by Giacomo [29] reported by using radial Basis probabilistic neural network classify the external defect of mango using hue and saturation histogram for ground region identification. Gray level co-occurrence matrixes for the quality of orange there are 400 samples of different defects like stabbing wounds, bruise, abrasion; sunburn, injury, and hail to damage are identified. The error rate of the system small it is $2.75 \%$

Internal defect detection in fruits by non-intrusive method by Anitha Raghavendra and Dr. Mahesh Rao [30] proposed the internal defects of fruits are very difficult process. This paper defined the identification of internal defects of fruits of the Post-harvest method. Here mango fruits internal defect detection described. Identify the physiological disorders, damage due to pests using Near Infrared Imaging (NIR).

In [24] inline function is used the author of the classification of Harumains mango. In here create platform for capturing of image with 2 mega pixel camera. Images are in RGB format, convert it to gray scale and apply multiple global thresholds to remove the background. Fill-holes algorithm is help to finding the dark sports and holes. To remove shadow 3 repeated global threshold applied first to B, then $\mathrm{G}$ again $\mathrm{B}$.To analyze the shape uses Fourier descriptor. Water displacement and disk methods are used for the evaluation of the volume.

Siti khair unniz bejo, et al,[26].( 2014) suggested sweetness of mango increased in ripening time. In here mango capturing using Keyence machine vision.AR 2008 refractometer is used for calculating the sweetness of the mango. The color is related to the sweetness of the mango fruit. The model builds on using Linear Regression. The average success of this system is $95.67 \%$
As described in [27], the identification of quality by using roundness and percent of defects Grabbed images RGB and $\mathrm{Cb}$ values are calculated. Selected optimal $\mathrm{Cb}$ value is referred as isodata.Thresholding process is used in here for finding the defective area.

In paper [19] fitness scaled chaotic artificial bee colony and ANN are the two types algorithms used digital camera used for the capturing and split and merge for background elimination. Using principal component analysis reduced the size of features; it covers $95 \%$ of variance between the original image .using histogram, found the pixels possible for gray scale value. For texture feature analysis users texture feature descriptor is used. It is the sum and difference of two random variables. Shape features extraction in 3 phases .first is using Euler number for finding perimeter and area. Second is Graham Sam method to create a convex hull. Construct an ellipse minor length, major length and eccentricity is the third step. FSCABC used for weight classification. The accuracy of the FSCABC-FNN is $89.1 \%$

\section{COMPARATIVE EVAUATION}

From this study we understand mainly KNN, ANN, fuzzy logics etc... are used for the classification. To improve the accuracy of the system, we can introduce new methods like Deep learning and Optimization .Also new features of the fruits may improve the efficiency of the system. Estimate the highlights and disadvantages of the fruits are in Table 1 shown below.

\section{CONCLUSION}

Agriculture is the basic food source of people in Kerala. From this work we have studied the different classification methods, maturity identification methods, defect detection methods used for fruit identification. In this study mainly ANN, SVM, KNN and Fuzzy logics are the classifiers used. From this one we understand the labor cost reduced using these works. Fuzzy logic gives less accuracy and ANN is most accurate method for classification. For the identification of maturity mainly color features are used. In the case of defect detection threshold gives the most accurate result. 
Table 1: comparison of approaches

\begin{tabular}{|c|c|c|c|c|}
\hline Sl. no & Task & Source & Highlights & Demerits \\
\hline 1 & Classification & $\begin{array}{l}\text { Mango size classification using RGB } \\
\text { color sensor and fuzzy logic(2016) } \\
\text { A.B Razak mansor et al. [11] }\end{array}$ & $\begin{array}{l}\text { Simple classification } \\
\text { Easy to develop } \\
\text { Accuracy } 85 \%\end{array}$ & Uncertainty \\
\hline 2 & Classification & $\begin{array}{l}\text { Mango fruit sortation system using NN } \\
\text { and computer vision(2017) } \\
\text { Emny Harna Yossy et al } \\
\text { [23 ] }\end{array}$ & $\begin{array}{l}\text { ANN predict the color } \\
\text { and size of the mango } \\
40 \text { hidden layers } \\
\text { Accuracy-94\% }\end{array}$ & $\begin{array}{l}\text { Training time is } \\
\text { higher than other } \\
\text { classifiers }\end{array}$ \\
\hline 3 & Classification & $\begin{array}{l}\text { Automatic fruit recognition and } \\
\text { counting from multiple images (2014). } \\
\text { Y. Song [18] }\end{array}$ & Use video processing & $\begin{array}{l}\text { The accuracy is lesser } \\
\text { than other classifier }\end{array}$ \\
\hline 4 & Defect detection & $\begin{array}{l}\text { Automatic classification of fruit defect } \\
\text { based on co-occurrence matrix and } \\
\text { Neural Network (2015) Giacomo et al. }\end{array}$ & $\begin{array}{l}\text { radial Basis } \\
\text { probabilistic neural } \\
\text { network classify the } \\
\text { external defect }\end{array}$ & High cost \\
\hline 5 & Defect detection & $\begin{array}{l}\text { Detection of external defects of } \\
\text { mango(2016) pujitha } \mathrm{N} \text { et al.[6] }\end{array}$ & $\begin{array}{l}\text { Grading based on } \\
\text { defected area } \\
\text { External defect } \\
\text { identified } \\
\text { Watershed algorithm }\end{array}$ & $\begin{array}{l}\text { Graphical results are } \\
\text { poor }\end{array}$ \\
\hline 6 & Grading & $\begin{array}{l}\text { Scientific classification of ripening } \\
\text { period and development of color grade } \\
\text { chart for Indian mangoes using } \\
\text { multivariant cluster analysis (2015) } \\
\text { V.Eyarkai Nambi et al.[14] }\end{array}$ & $\begin{array}{l}5 \text { grade classification } \\
\mathrm{L}^{*} \mathrm{a} \mathrm{b}^{*} \text { color format } \\
\text { Acidity of mango } \\
\text { decrease up to 16th day }\end{array}$ & $\begin{array}{l}\text { Machine sensitivity } \\
\text { low } \\
\text { Need expert labors }\end{array}$ \\
\hline 7 & $\begin{array}{l}\text { Maturity } \\
\text { identification }\end{array}$ & $\begin{array}{l}\text { Defect maturity of permission fruit } \\
\text { based on image processing(2014) } \\
\text { Vahid Mohammadi et al.[21] }\end{array}$ & $\begin{array}{l}\text { Use SPSS software for } \\
\text { analysis of variance } \\
\text { Accuracy is } 90.2 \\
\text { Using QAD and LDA }\end{array}$ & $\begin{array}{l}\text { Graphical results are } \\
\text { poor }\end{array}$ \\
\hline
\end{tabular}

\section{REFERENCES}

[1] Dameshwari sahu and Ravindra Manohar Potdar,Defect identification and maturity detection of mango fruits using image processing. International Journal of Scientific Research in Computer Science, Engineering and Information Technology.2017.

[2] Tabbu Mulani,Shahin Khan et al. An Automated Method based on Image Processing for Grading of Harvested Mangoes.International Journal of Scientific Research in Computer Science, Engineering and Information Technology.2017.

[3] Dhanashree Jawale and Manjusha Deshmukh.2017. Real Time Automatic Bruise Detection in (Apple) Fruits Using Thermal Camera International Conference on Communication and Signal Processing

[4] Mohammed A. H. Ali and Kelvin Wong Thai.2017. Automated Fruit Grading System.IEEE 3rd International Symposium on Robotics and Manufacturing Automation (ROMA)

[5] M. Sahana and H. B. Anita.2017. Automatic Classification of South Indian Regional Fruits using Image Processing.Indian Journal of Science and Technology
[6] Pujitha N, Swathi C and Kanchana V. Detection of External Defects on Mango.International Journal of Applied Engineering Research ISSN 0973-4562.2017.

[7] Misigo Ronald, Miriti Evans. Classification of selected (apple) fruit varieties using naive bayes.Indian Journal of Computer Science and Engineering (IJCSE).2016

[8] Yoshio Makino,Kenjiro Goto et al. A Grading Method for Mangoes on the Basis of Peel Color Measurement Using Computer Vision System.Agricultural Sciences.2016.

[9] Yudong Zhang a,Shuihua Wang at el. Fruit classification using computer vision and feedforward neural network. ELSEVIER.2016.

[10] MS Seena and PM mahajan.2016.A fruit detecting and grading system based on imageprocessing.IJIREEICE2016

[11] Ab Razak Mansor, Mahmod Othman et al.Mango Size Classification Using RGB Color Sensor and Fuzzy Logic Technique. Springer Science+Business Media Singapore 2016

[12] F.S.A. Sa'ad , M.F. Ibrahim A at el. Shape and weight grading of mangoes using visible imaging.ELSEVIER.2015.

[13] Seema, A kumar and G.S Gill.2015. Automatic Fruit Grading and Classification System Using Computer Vision 
[14] V.Eyarkai Nambi, K. Thangavel and D.Manohar Jesudas. Scientific classification of ripening period and development of color grade chart for Indian mangoes (Mangifera indica L.) using multivariate cluster analysis. ELSEVIER.2015.

[15] Sapan Naik, Bankim Pate and, Rashmi Pandey.2015. Shape, size and maturity features extraction with fuzzy classifier for non-destructive mango (Mangifera Indica L., cv. Kesar) grading.2015-IEEE International Conference on Technological Innovations in ICT for Agriculture and Rural Development (TIAR 2015)

[16] Ramya M and Anitha Raghavendra. Ripeness Evaluation of Mangoes using Image Processing.IJESC.2015.

[17] Ghulam Muhammad.. Date fruits classification using texture descriptors and shape-size features. ELSEVIER.2014.

[18] Y. Song, C.A. Glasbey, G.W. Horgan et al. Automatic fruit recognition and counting from multiple images. ELSEVIER

[19] Yu-Dong Zhang,Genlin ji at elFruit classification using computer vision and feed forward Neural Network. ELSEVIER.2014.

[20] Chandra Sekhar Nandi, Bipan Tudu, and Chiranjib Koley.2014. Computer Vision Based Mango Fruit Grading System. International conference on Innovative Engineering Technologies (ICIET'2014)

[21] Vahid Mohammadi, Kamran Kheiralipour et al. Detecting maturity of persimmon fruit based on image processing technique.ELSEVIER.2014.

[22] Jang-yoon Kim, Michael Vogl, Shin-Dug Kim.2014. A Code based Fruit Recognition Method Via Image Convertion Using Multiple Features.IEEE

[23] Emny Harna Yossya, Jhonny Pranataa at el.Mango Fruit Sortation System using Neural Network and Computer Vision. ELSEVIER.2014.
[24] Mohd Firdaus Ibrahim, Fathinul Syahir Ahmad Sa'ad et al.2016. In-Line Sorting of Harumanis Mango Based on External Quality Using Visible Imaging.senser

[25] Rashmi Pandey,Nikunj Gamit and Sapan Naik.2014. NonDestructive Quality Grading Of Mango (Mangifera Indica L) Based On CIELAB Colour Model and Size.2014 -IEEE International Conference on Advanced Communication Control and Computing Technologies (ICACCCT)

[26] Siti Khairunniza Bejo and Syahidah Kamaruddin.2014 Determination of Chokanan mango sweetness (Mangifera indica) using non-destructive image processing technique.AJCS.

[27] Tomas U. Ganiron Jr.Size Properties of Mangoes using Image Analysis.International Journal of Bio-Science and Bio-Technology, principles, developments and applications of computer vision for external quality inspection of fruits and vegetables.2014.

[28] Baohua Zhang,Wenqian Huang et al.2014.Principles, developments and applications of computer vision for external quality inspection of fruits and vegetables

[29] Giacomo Capizzi,Grazia Lo Sciuto et al.2015.Automatic Classification of Fruit Defects based on Co-occurrence Matrix and Neural Networks.Proceedings of the Federated Conference on Computer Science and Information Systems

[30] Anitha Raghavendra and Dr. Mahesh rao. A Survey on Internal Defect Detection in Fruits by Non-Intrusive Method. International journal of latest trends in Engineering and technology.2014.

[31] Sapan Naik and Bankim Patel .2017.Thermal imaging with fuzzy classifier for maturity and size based nondestructive Mango (Mangifera Indica L.) grading.2017International Conference on Emerging Trends \& Innovation in ICT (ICEI) 